

\title{
Biodiversidad bacteriana en un riachuelo con vertido continuo de efluentes de hidrocarburos
}

\author{
Marelis M. Ruiz ${ }^{*}(\mathbb{D})$, Elen B. de Souza Carvalho ${ }^{2}$ (D) Bruno C. Ladeira ${ }^{3}$ y Marcelo A. de Oliveira ${ }^{4}$ (D) \\ ${ }^{1}$ Facultad de Ingeniería, Escuela de Industrial, Universidad del Zulia, Maracaibo, C.P 4001, Venezuela \\ ${ }^{2}$ Laboratorio de Tecnología de DNA, Universidad Federal do Amazonas UFAM, Manaos-AM, C.P 69067-005, \\ Brasil \\ ${ }^{3}$ Petrobras (UN-AM), Gerencia de Seguridad, Medio Ambiente y Salud. C.P 69055-035, Manaos, Brasil \\ ${ }^{4}$ Facultad de Tecnología-Departamento de Ingeniería de Producción, Universidad Federal del Amazonas UFAM, \\ Manaos-AM, C.P 69080-900, Brasil \\ *Autor de correspondencia: marelis_ruiz@yahoo.es \\ https://doi.org/10.22209/rt.v44n2a01
}

Recepción: 13 de abril de 2020 | Aceptación: 12 de diciembre de 2020 | Publicación: 01 de abril de 2021

\section{Resumen}

Desde el inicio de sus operaciones, la provincia petrolera de Urucu (Petrobras unidad Amazonas, Brasil, UN-AM) ha realizado el vertido continuo de efluentes de hidrocarburos en riachuelos alrededor de sus instalaciones. El objetivo de esta investigación fue realizar un análisis de la comunidad bacteriana existente en un riachuelo (comunidad 1: antes del vertido, comunidad 2: después del vertido). Se colectaron muestras de agua en riachuelo para extraer el DNA genómico total y usarlo como molde en la PCR, con oligonucleótidos específicos del gen 16S rDNA para dominio Bacteria. El producto de la PCR fue amplificado, y las secuencias generadas por técnica de pirosecuenciación, fueron analizadas con el programa libre Mothur. Los resultados revelaron que el filo Acidobacteria, la clase Deltaproteobacteria y los géneros Gp3 y Geobacter, mostraron alta presencia en comunidad 1. Asimismo, el filo Proteobacteria fue el más abundante, con la clase Betaproteobacteria y los géneros Geobacter y Gp3, como los predominantes en comunidad 2. Los índices de riqueza presentaron variación porcentual en ambas comunidades (disminución e incremento), siendo la diversidad más abundante en la comunidad 1 . Sin embargo, no se encontró diferencia significativa entre ambas comunidades $(p>0,001)$, y todas muestras de cada comunidad presentaron la misma estructura genética $(p<0,05)$. El $25 \%$ de los géneros bacterianos se consideraron "no clasificados", y aproximadamente el $15 \%$ de los géneros fueron clasificados como biorremediadores. Estos datos representan un desafío para la exploración biotecnológica en este ecosistema, con potencial para identificar y clasificar nuevos grupos taxonómicos. diversidad.

Palabras clave: actividad petrolera, Amazonia, análisis metagenómico, gen 16S rDNA, índices de riqueza y

\section{Bacterial biodiversity analysis in a stream with continuous disposal of hydrocarbon effluents}

\begin{abstract}
Since the beginning of its operations, the Urucu petroleum province (Petrobras, Amazonas unit - AM, Brazil) has been continuously discarding industrial wastewater from an effluent dike in a small water stream outside of the perimeter of its operational area. The objective of this research was to perform a study of the bacterial community existent in a small water stream (community 1: prior to effluent disposal, community 2: after effluent disposal) Water samples from the stream were collected to totally extract their genomic DNA and subsequently utilize it as a template in the PCR using specific oligonucleotides of 16S rDNA gene from the Bacteria domain. The PCR product was amplified, and the sequences obtained through a pyrosequencing method were analyzed using the free software Mothur. The results obtained from the software revealed the Acidobacteria phylum, Deltaproteobacteria class, and the Gp3 and Geobacter genus were all preponderant in community 1. Likewise, in community 2, the Proteobacteria phylum, Betaproteobacteria class, and the Gp3 and Geobacter genus were preponderant. The richness indexes reported a percentage change (both decrease and increase) for both
\end{abstract}


communities; furthermore, these indicated that the diversity was more abundant in community 1 . However, no significant variance was observed between both communities $(p>0.001)$ and all samples from each community yielded identical genetic structures $(p<0.05)$. The $25 \%$ of the bacterial genders were cataloged as "unclassified", and approximately $15 \%$ of the genders were cataloged as bio-remediators. The aforementioned data represents a challenge for biotechnological exploration in this environment, with the potential to identifying and classifying new taxonomic groups.

Keywords: Amazon, metagenomic study, oil activity, richness and diversity indexes, 16S rDNA gene.

\section{Introducción}

Una gran parte de la biodiversidad en los ecosistemas amazónicos está representada por hongos y bacterias. En las muestras ambientales, la estructura de las comunidades bacterianas es altamente compleja y diversa (Torsvik et al., 1990), y esta complejidad representa un desafío para la biotecnología. Con respecto a los microorganismos biorremediadores de ambientes contaminados, los mismos se encuentran ampliamente distribuidos en el suelo y cuerpos de agua, cuyas comunidades normalmente constituyen menos que 1\% de la comunidad bacteriana total, pero cuando los hidrocarburos están presentes, estas comunidades aumentan en 10\% (Atlas y Cerniglia, 1995). Ciertas estimaciones indican que aproximadamente $99 \%$ de los microorganismos presentes en muchos ambientes naturales, no son cultivables en laboratorio mediante técnicas tradicionales de cultivo (Amann et al., 1995). Los avances recientes en biología molecular, especialmente en la tecnología de secuenciación del ácido desoxirribonucleico (DNA, según sus siglas en inglés), han brindado más oportunidades para estudios exhaustivos de microorganismos en diversos ecosistemas (Parmar et al., 2019), específicamente realizando análisis metagenómico (genomas secuenciados directamente de la muestra ambiental, sin necesidad de cultivo y aislamiento de microorganismos en laboratorio), para determinar de manera más realista la diversidad bacteriana (Peixoto et al., 2011).

En medio de la región amazónica brasileña, la provincia petrolera de Urucu constituye el único activo de producción de petróleo y gas de la empresa Petrobras (unidad Amazonas UN-AM). Desde el inicio de sus operaciones en el año 1988, los efluentes industriales de esta provincia petrolera, son vertidos en pequeños riachuelos alrededor del área operacional, desde un dique de efluentes. Esto después de realizar el control en la estación de tratamiento de efluentes (ETE), validando que los parámetros fisicoquímicos estén dentro de la normativa ambiental del Consejo Nacional del Medio Ambiente, específicamente la norma № 357 (marzo de 2005) (CONAMA, 2005).

A pesar del cumplimiento de la normativa ambiental para el vertido de efluentes en cuerpos de agua de la provincia petrolera de Urucu, la empresa Petrobras (UN-AM) no ha realizado una evaluación de los microorganismos existentes en el ecosistema del riachuelo donde se vierten los efluentes desde el inicio de sus operaciones, lo cual es fundamental para conocer la biodiversidad (análisis taxonómico) y garantizar la conservación del mismo. La taxonomía es la ciencia que identifica una especie, la cual es un grupo de individuos (especímenes) que muestran, en mayor o menor grado, la variabilidad intrapoblacional siempre presente (Bicudo, 2004). Entre las diferentes herramientas tecnológicas para realizar análisis de taxonomía, determinación de índices de riqueza y diversidad, así como la diferencia estadística entre las comunidades de estudio, se encuentra el programa libre Mothur (Schloss, 2009).

El objetivo de esta investigación fue realizar un análisis de la comunidad bacteriana existente en un riachuelo (comunidad 1: antes del vertido, comunidad 2: después del vertido), donde es realizado el vertido continuo de efluentes por la empresa Petrobras (unidad Amazonas UN-AM), para identificar taxonómicamente los microorganismos y comparar estadísticamente la riqueza y diversidad existente en este ecosistema.

\section{Materiales y Métodos}

\section{Área de estudio}

El estudio fue desarrollado en la provincia petrolera de Urucu, denominada base operacional de Petrobras geólogo

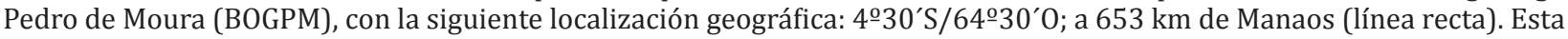
base se encuentra en la cuenca del río Urucu en el margen derecho del río Solimões, en el municipio Coari, estado Amazonas, Brasil.

\section{Parte experimental}

Después de varias visitas al campo en la provincia petrolera de Urucu (Petrobras UN-AM), en septiembre de 2011 se realizó la recolección de muestras para esta investigación. Fueron seleccionados ocho puntos de colecta (4 puntos aguas arriba del vertido y 4 puntos aguas abajo del vertido de efluentes), recaudando en total $16 \mathrm{l}$ de agua clasificadas como 
comunidad 1 (riachuelo antes de vertido) y comunidad 2 (riachuelo después del vertido), y depositados en botellas de plástico esterilizadas, siendo $2 \mathrm{l}$ por cada punto. Los datos y coordenadas geográficas de todos los puntos de colecta de muestras, se encuentran en la Tabla 1.

En el laboratorio, las muestras fueron centrifugadas en tubos Falcon de $50 \mathrm{ml}$, descartando el agua del sobrenadante, hasta obtener apenas el material sólido (mínimo de 0,25 g requeridos para cada extracción de DNA). Para este proceso se utilizó un centrifugador marca Eppendorf modelo 5810R, a una rotación de 4.000 rpm durante 10 min. Para la extracción del DNA total se siguió el procedimiento recomendado por el fabricante del PowerSoilTM DNA Isolation Kit (MoBio Laboratories Inc., EE.UU.), a fin de realizar el estudio metagenómico de las muestras.

Tabla 1. Localización de puntos de colecta de muestras de agua en el riachuelo con vertido continuo de efluentes de hidrocarburos.

\begin{tabular}{|c|c|c|c|c|}
\hline $\mathbf{N}^{\circ}$ & Lugar & Código & $\begin{array}{c}\text { Coordenadas } \\
\text { geográficas }\end{array}$ & $\begin{array}{c}\text { Profundidad de la } \\
\text { colecta (m) }\end{array}$ \\
\hline 1 & $\begin{array}{l}\text { Centro de riachuelo a } 50 \mathrm{~m} \\
\text { aguas arriba }\end{array}$ & $\mathrm{P} 1$ & $\begin{array}{l}\mathrm{S} 04^{\circ} 51^{\prime} 40,4^{\prime \prime} \\
\mathrm{O} 065^{\circ} 17^{\prime} 52,7^{\prime \prime}\end{array}$ & 0,2 \\
\hline 2 & Orilla de riachuelo a $50 \mathrm{~m}$ aguas arriba & P2 & $\begin{array}{l}\text { S } 04^{\circ} 51^{\prime} 40,4^{\prime \prime} \\
\text { O } 065^{\circ} 17^{\prime} 52,7^{\prime \prime}\end{array}$ & 0,2 \\
\hline 3 & Centro de riachuelo a $80 \mathrm{~m}$ aguas arriba & P3 & $\begin{array}{l}\mathrm{S} 04^{\circ} 51^{\prime} 40,3^{\prime \prime} \\
\mathrm{O} 065^{\circ} 17^{\prime} 52,8^{\prime \prime}\end{array}$ & 0,3 \\
\hline 4 & Orilla de riachuelo a $80 \mathrm{~m}$ aguas arriba & P4 & $\begin{array}{l}\mathrm{S} 04^{\circ} 51^{\prime} 40,3^{\prime \prime} \\
\mathrm{O} 065^{\circ} 17^{\prime} 52,8^{\prime \prime}\end{array}$ & 0,2 \\
\hline 5 & $\begin{array}{l}\text { Centro de riachuelo a } 50 \mathrm{~m} \\
\text { aguas abajo }\end{array}$ & P5 & $\begin{array}{l}\mathrm{S} 04^{\circ} 51^{\prime} 40,8^{\prime \prime} \\
\mathrm{O} 065^{\circ} 17^{\prime} 50,8^{\prime \prime}\end{array}$ & 0,3 \\
\hline 6 & $\begin{array}{l}\text { Orilla de riachuelo a } 50 \mathrm{~m} \\
\text { aguas abajo }\end{array}$ & P6 & $\begin{array}{l}\mathrm{S} 04^{\circ} 51^{\prime} 40,8^{\prime \prime} \\
\mathrm{O} 065^{\circ} 17^{\prime} 50,8^{\prime \prime}\end{array}$ & 0,2 \\
\hline 7 & $\begin{array}{l}\text { Centro de riachuelo a } 80 \mathrm{~m} \\
\text { aguas abajo }\end{array}$ & P7 & $\begin{array}{l}\mathrm{S} 04^{\circ} 51^{\prime} 40,9^{\prime \prime} \\
\mathrm{O} 065^{\circ} 17^{\prime} 50,4^{\prime \prime}\end{array}$ & 0,2 \\
\hline 8 & $\begin{array}{l}\text { Orilla de riachuelo a } 80 \mathrm{~m} \\
\text { aguas abajo }\end{array}$ & P8 & $\begin{array}{c}\mathrm{S} 04^{\circ} 51^{\prime} 40,9^{\prime \prime} \\
\mathrm{O} 065^{\circ} 17^{\prime} 50,3^{\prime \prime}\end{array}$ & 0,2 \\
\hline
\end{tabular}

Posteriormente, el material fue colocado en un termociclador (AB Applied Biosystems veriti 96 well Thermol cycler) programado para realizar el siguiente ciclo de reacción en cadena de la polimerasa (PCR, según sus siglas en inglés): desnaturalización inicial a $95{ }^{\circ} \mathrm{C}$ por $4 \mathrm{~min}, 35$ ciclos de desnaturalización a $95{ }^{\circ} \mathrm{C}$ por 1 min, anillamiento a 59 o $\mathrm{C}$ por 1 min y extensión a $72{ }^{\circ} \mathrm{C}$ por $40 \mathrm{seg}$; seguida de extensión final a $72{ }^{\circ} \mathrm{C}$ por $5 \mathrm{~min}$. Se utilizaron cuatro tipos de iniciadores (primers): MID1, MID2, MID3 y MID4, combinando todas las muestras con cada uno de estos, para luego ser corridos en dos plataformas diferentes, utilizando la técnica de pirosecuenciación (454 Roche). manera:

Para facilitar el análisis posterior, las muestras fueron agrupadas en dos comunidades, catalogadas de la siguiente

- Comunidad 1: conformado por las muestras P1, P2, P3 y P4.

- Comunidad 2: conformado por las muestras P5, P6, P7 y P8.

La mezcla para PCR fue compuesta por $5 \mu$ l de DNA de cada muestra, $1 \mu$ l del primer iniciador, $1 \mu \mathrm{l}$ del iniciador reverso, enzima taq DNA polimerasa y agua ultra pura Milli-Q (agua desionizada) para completar una solución final de 25 $\mu \mathrm{l}$. La PCR fue realizada por quintuplicado para las ocho muestras, y la combinación de los iniciadores con las diferentes muestras o bibliotecas, fue la siguiente (la especificación de los iniciadores se encuentra descrita en la Tabla 2): 
- Muestra 1: P1 con MID1
- Muestra 2: P2 con MID2
- Muestra 3: P3 con MID3
- Muestra 4: P4 con MID4
- Muestra 5: P5 con MID1

- Muestra 6: P6 con MID2

- Muestra 7: P7 con MID3

- Muestra 8: P8 con MID4

Tabla 2. Iniciadores utilizados en la amplificación de fragmentos de DNA para las comunidades del riachuelo con vertido continuo de efluentes de hidrocarburos.

\begin{tabular}{ccl}
\hline Tipo & Especificación & \multicolumn{1}{c}{ Secuencia de nucleótidos (5'-3') } \\
\hline $\begin{array}{c}\text { Iniciador } \\
\text { reverso } \\
\text { (primer } \\
\text { reverse })\end{array}$ & Lib-L B Key & $\begin{array}{l}\text { CCTATCCCCTGTGTGCCTTGGCAGTCTCAGGGGACTACCAGGG } \\
\text { TATCTAAT }\end{array}$ \\
& & \\
& 16S-LibL-F-MID1 & $\begin{array}{l}\text { CCATCTCATCCCTGCGTGTCTCCGACTCAGACACGACGACTAC } \\
\text { TCCTACGGRAGGCAGCAG }\end{array}$ \\
Iniciadores & 16S-LibL-F-MID2 & $\begin{array}{l}\text { CCATCTCATCCCTGCGTGTCTCCGACTCAGACACGTAGTATACT } \\
\text { CCTACGRAGGCAGCAG }\end{array}$ \\
frimers & 16S-LibL-F-MID3 & $\begin{array}{l}\text { CCATCTCATCCCTGCGTGTCTCCGACTCAGACACTACTCGTACT } \\
\text { CCTACGGRAGGCAGCAG }\end{array}$ \\
& & CCATCTCATCCCTGCGTGTCTCCGACTCAGACGACACGTATACT \\
& CCTACGGRAGGCAGCAG \\
\hline
\end{tabular}

El proceso de purificación del DNA se realizó utilizando el GFXTM PCR DNA and gel band purification kit (GE Healthcare), siguiendo el procedimiento indicado por el fabricante (corte de las bandas en faja de 500 pares de base pb). Con el objetivo de validar la purificación de las muestras, se realizó una electroforesis en gel de agarosa 0,8 \% y marcador de peso molecular $1 \mathrm{~kb}(1 \mathrm{~kb}=1.000$ pares de bases) como referencia, $5 \mu \mathrm{l}$ de cada muestra purificada, aproximadamente por $1 \mathrm{~h}$ a $70 \mathrm{volts}$.

Los productos finales de cada tratamiento se almacenaron a una temperatura criogénica de $-20{ }^{\circ} \mathrm{C}$, hasta ser enviados a la empresa GenOne (Rio de Janeiro-RJ, Brasil), para aplicar la técnica de Pirosecuenciación (plataforma Roche 454) y obtener resultados íntegros de alta calidad en el estudio metagenómico, aplicando el programa libre Mothur (versión 1.34.0, febrero 2014) (Schloss, 2009).

\section{Resultados y Discusión}

Como resultado de la aplicación de la técnica de Pirosecuenciación (Roche 454), se obtuvieron secuencias de nucleótidos para todas las muestras consideradas en el presente estudio, las cuales fueron analizadas mediante el programa libre Mothur (Schloss, 2009).

\section{Clasificación taxonómica}

La identificación y clasificación taxonómica para esta investigación, fue basada en el programa libre Mothur. El análisis taxonómico fue realizado a nivel de: filo, clase y género.

Filos

Se encontraron 28 filos bacterianos catalogados científicamente, así como también filos "no clasificados" (microorganismos no catalogados científicamente). La Figura 1 presenta la proporción de los filos predominantes, con 
valores de frecuencia relativa $>0,1 \%$ en alguna muestra.

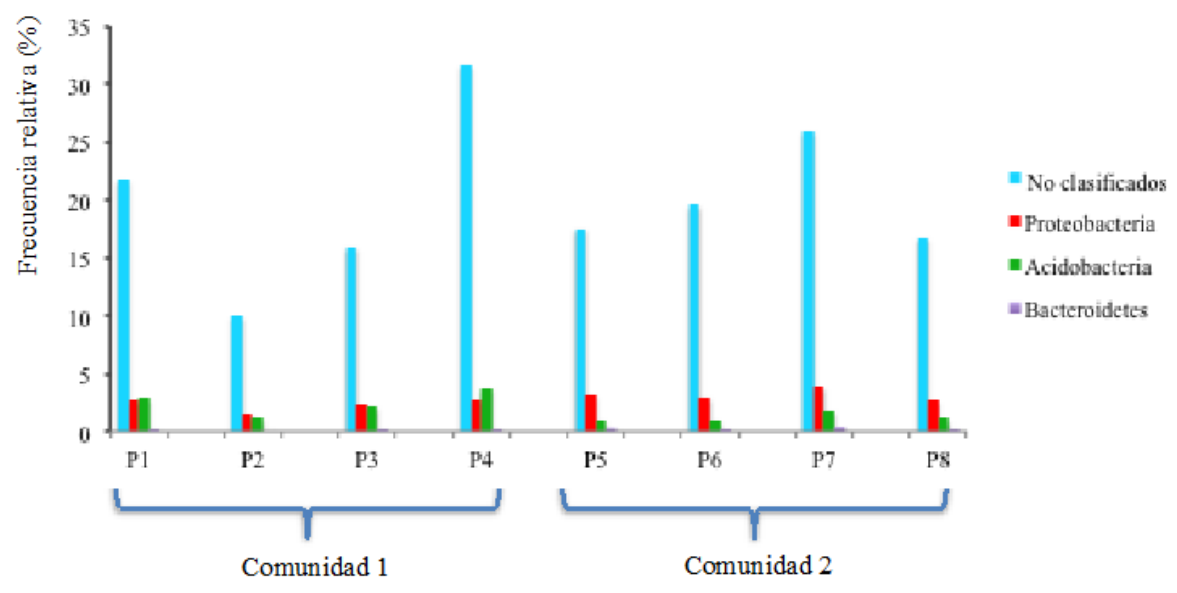

Figura 1. Filos bacterianos predominantes en las comunidades del riachuelo con vertido continuo de efluentes de hidrocarburos. Comunidad 1: antes del vertido, comunidad 2: después del vertido.

Los filos predominantes en las dos comunidades fueron de tipo "no clasificados" ( $>30 \%$ de frecuencia relativa), seguido de los filos Proteobacteria y Acidobacteria ( $<5 \%)$. Se pudo observar un incremento en el filo Proteobacteria en la comunidad 2, resultando un valor máximo de frecuencia $<4 \%$; diferente de la comunidad 1 , que tuvo valor máximo de frecuencia $<3 \%$. Con relación al filo Acidobacteria, sucedió lo contrario, la magnitud de frecuencia disminuyó en la comunidad $2(<2 \%)$, mientras que en la comunidad 1, el valor máximo de frecuencia alcanzado fue de $<4 \%$.

En un estudio realizado para analizar la diversidad microbiana presente en el río Negro (afluente del Amazonas, Brasil), se identificó como filo predominante (81 \%) Proteobacteria (Neves, 2013). Igualmente, el filo Proteobacteria fue el más abundante en todas las muestras de ríos y lagos de la región amazónica de Brasil (Peixoto et al., 2011; Rodrigues, 2011; Toyama, 2012), el cual es característico de ambientes de agua dulce (Zwart et al., 2002).

\section{Clases}

Se identificaron 40 clases bacterianas catalogadas científicamente y otras desconocidas (no clasificadas). En la Figura 2, se presenta la distribución de clases predominantes en las dos comunidades de estudio.

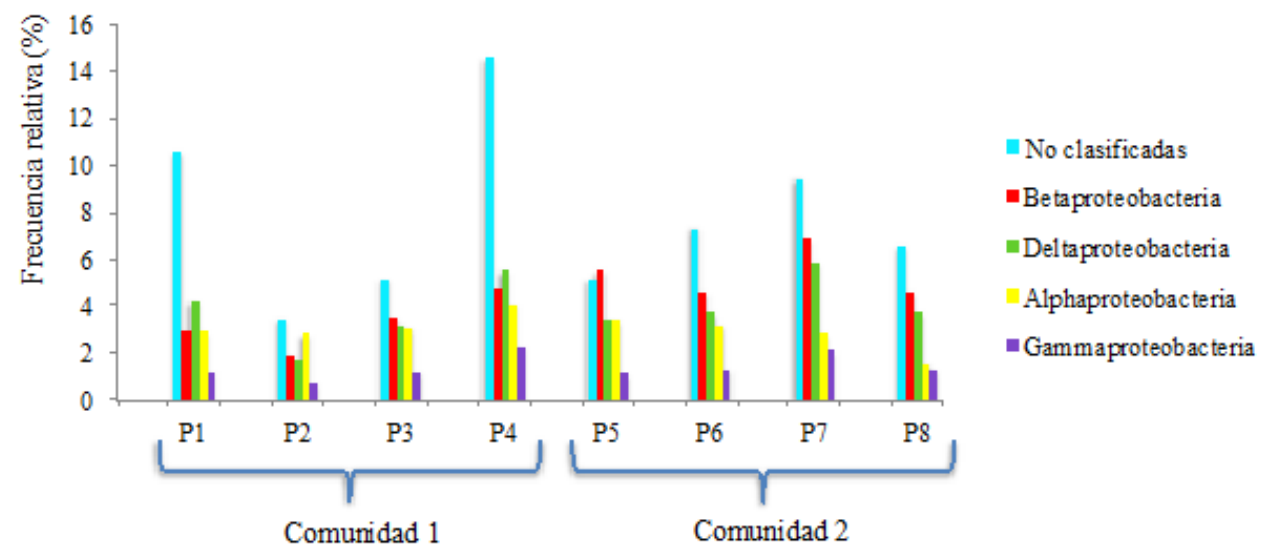

Figura 2. Clases bacterianas predominantes en las comunidades del riachuelo con vertido continuo de efluentes de hidrocarburos. Comunidad 1: antes del vertido, comunidad 2: después del vertido.

Las clases con mayor frecuencia relativa en las dos comunidades fueron las "no clasificadas" ( $<15 \%)$. En la comunidad 1 la clase dominante fue Deltaproteobacteria $(<6 \%)$, seguida de la clase Betaproteobacteria $(<5 \%)$. Específicamente en la 
comunidad 2, se observó una predominancia en la frecuencia de la clase Betaproteobacteria $(<7 \%)$, seguida de la clase Deltaproteobacteria $(<6 \%)$ y la clase Alphaproteobacteria en tercer lugar $(<4 \%)$. La clase Alphaproteobacteria no presentó variabilidad significativa en las dos comunidades $(<5 \%)$.

Con relación al contexto anterior, Peixoto et al. (2011) obtuvieron la clase Betaproteobacteria como la más predominante en el río Negro y la Betaproteobacteria en el río Solimões (afluentes del rio Amazonas, Brasil). También Rodrigues (2011) encontró como preponderante la clase Alphaproteobacteria en el estuario del río Amazonas.

\section{Géneros}

Se identificaron 267 géneros bacterianos, además de los "no clasificados". En la Figura 3 pueden observarse los tipos de géneros dominantes, sin incluir los géneros aún no clasificados, a fin de facilitar la visualización gráfica.

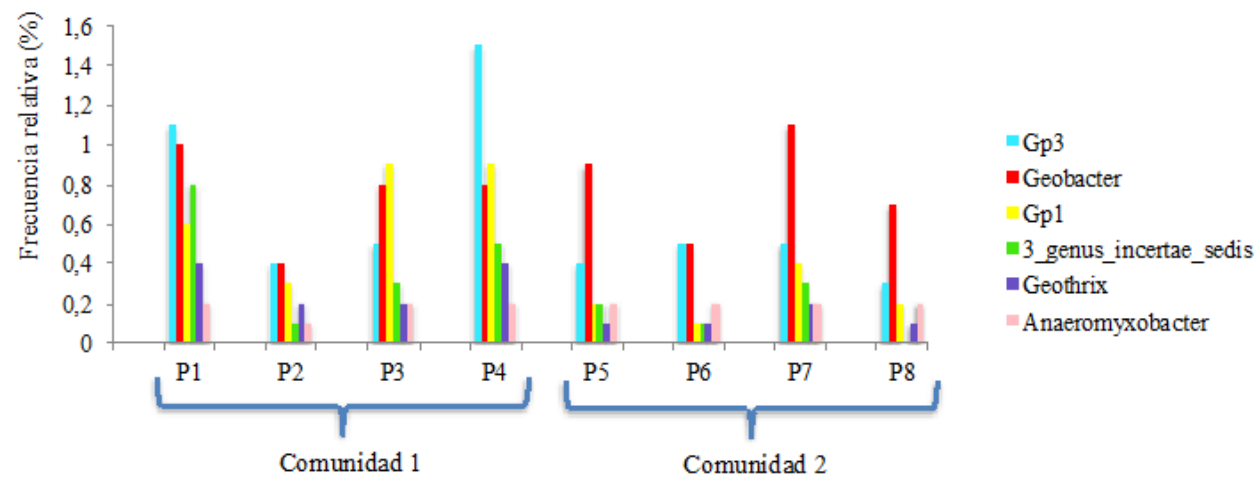

Figura 3. Géneros bacterianos predominantes (excepto los "no clasificados") en las comunidades del riachuelo con vertido continuo de efluentes de hidrocarburos. Comunidad 1: antes del vertido, comunidad 2: después del vertido.

La comunidad 1 exhibió la mayor frecuencia de géneros bacterianos, resultando como más dominantes los géneros Gp3 (<1,6 \%), Geobacter y Gp1 (ambos <1 \%) y 3_genus_incertae_cedis ( $<0,8 \%)$. Luego, en menor proporción $(<0,2 \%)$, siguieron los géneros Geotrix y Anaeromixobacter. Los géneros que presentaron porcentaje de frecuencia relativa muy bajo, no son mostrados en este gráfico.

En la comunidad 2, los géneros Geobacter $(<1,2 \%)$ y Gp3 $(<0,5 \%)$ fueron los más preponderantes, seguidos del Gp1 (<0,4 \%), el 3_genus_incertae_sedis, Anaeromyxobacter y Geothrix (todos <0,3 \%).

Para una mejor visualización gráfica, los géneros bacterianos "no clasificados" son presentados en la Figura 4. Se puede observar que la mayor proporción de los géneros todavía no catalogados, se encuentra en la comunidad 1 ( $<31 \%$ ), seguida de la comunidad de la comunidad $2(\leq 25 \%)$.

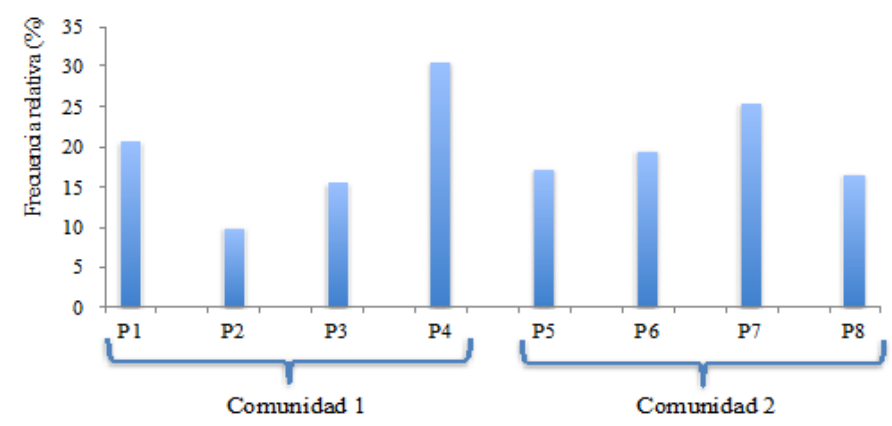

Figura 4. Proporción de géneros bacterianos "no clasificados" en las comunidades del riachuelo con vertido continuo de efluentes de hidrocarburos. Comunidad 1: antes del vertido, comunidad 2: después del vertido. 
El género Gp3 pertenece al filo Acidobacteria y la clase Acidobacteria_Gp3, el cual también fue identificado en el estudio realizado por Neves (2013) en el río Negro (<11\%). El género Geobacter, que corresponde al filo Proteobacteria y la clase Deltaproteobacteria, es estrictamente anaeróbico y capaz de degradar diversos compuestos orgánicos y aromáticos (Kleinsteuber et al., 2012). Igualmente, el género Geobacter fue identificado con una frecuencia de 0,07 \% en el río Negro (Neves, 2013). También está el caso de estudio de la comunidad microbiana del río Grangeiro, en el cual el género Geobacter presentó una frecuencia relativa aproximada de $41 \%$ de genes resistentes al zinc (Xavier et al., 2019).

El género 3_genus_incertae_sedis pertenece al filo Verrucomicrobia y a la clase subdivision 3. El filo Verrucomicrobia representa una fracción significativa de las comunidades de agua dulce (Nishimura y Nagata, 2007), sin embargo, también representa entre $2-8 \%$ de la comunidad bacteriana observada en la rizosfera (Kielak et al., 2008). El género Geothrix que pertenece al filo Acidobacteria y a la clase Holophagae, es un organismo estrictamente anaeróbico con capacidad de reducción de hierro (Nevin y Lovley, 2002) y nitrato (Jin et al., 2015). Cannavan (2007) analizó la diversidad bacteriana en suelos de la región amazónica, y las secuencias presentaron valores menores de 31 \% de frecuencia en el género Geothrix.

Con relación al género Anaeoromyxobacter, se define como un microorganismo estrictamente anaeróbico y capaz de degradar sustratos orgánicos (Sun et al., 2012). Estos microorganismos fueron observados en ambientes con alta carga orgánica y tienen regularmente participación en las reacciones de hidrólisis de compuestos orgánicos complejos (Hatamono, 2007; Pereyra, 2010). Ramos (2013) identificó este microorganismo, específicamente una subespecie Anaeromyxobacter spp., en la caracterización de aguas subterráneas contaminadas con mezcla de diesel y biodiesel, con una frecuencia relativa de aproximadamente 18 \%. Igualmente, Silva et al. (2007) identificaron el género Anaeoromyxobacter en muestras de manglar de una cuenca petrolera (5\%).

En un estudio realizado en ambientes contaminados con metales pesados, de igual manera, el género Anaeoromyxobacter fue uno de los predominantes en el riachuelo Los Macacos, con frecuencia relativa aproximada de 38 \% (Xavier, 2019). El genoma completo de Anaeromyxobacter sp. Fw109-5 (bacteria reductora de metal), fue aislado de un medio contaminado, demostrando un gran potencial para procesos de biorremediación (Hwang, 2015).

El género Gp1 pertenece al filo Acidobacteria y la clase Acidobacteria_Gp1, y fue identificado por Etto (2011) en una comunidad de turfeiras (plantas pertenecientes a la familia Gentianaceae), y también por Ferreira (2011) como el género preponderante en el ambiente acuático donde se desarrolla una planta carnívora.

Del total de los 267 géneros bacterianos identificados, el $15 \%$ de estos microorganismos fueron catalogados como "biorremediadores" de ambientes contaminados, según lo relatado por varios autores (Mandri y Lin, 2007; Jacques et al., 2007; Alvarado, 2009; Seo et al., 2009; Nústez et al., 2014; Xavier, 2019). Asimismo, estos microorganismos biorremediadores presentan incremento en el número de secuencias finales, triplicando el valor en algunos casos específicos; corroborando que cuando los hidrocarburos están presentes, estas comunidades aumentan en $10 \%$ de la comunidad total (Atlas y Cerniglia, 1995).

\section{Curva de rarefacción e índices de riqueza y diversidad}

El agrupamiento de conjuntos de especies o géneros similares se denomina unidad taxonómica operativa (OTU, según sus siglas en inglés), los cual es la base para calcular las curvas de rarefacción, los índices de riqueza y diversidad de los microorganismos existentes en ecosistema estudiado. En la Tabla 3, se encuentran los datos de números de secuencias (inicial, final y no clasificados), así como la distribución de OTU entre las diferentes muestras.

Tabla 3. Distribución de OTU para las comunidades del riachuelo con vertido continuo de efluentes de hidrocarburos. Comunidad 1: antes del vertido, comunidad 2: después del vertido.

\begin{tabular}{cccccc}
\hline $\begin{array}{c}\text { Número de } \\
\text { comunidad }\end{array}$ & $\begin{array}{c}\text { Código } \\
\text { muestra }\end{array}$ & $\begin{array}{c}\mathbf{N}^{\circ} \text { secuencias } \\
\text { inicial }\end{array}$ & $\begin{array}{c}\mathbf{N}^{\circ} \text { secuencias } \\
\text { final }\end{array}$ & $\begin{array}{c}\text { No secuencias } \\
\text { no clasificadas }\end{array}$ & $\mathbf{N}^{\circ}$ OTU \\
\hline & P1 & 15.762 & 10.000 & 6.682 & 2.578 \\
1 & P2 & 6.969 & 4.000 & 2.691 & 1.109 \\
& P3 & 18.652 & 13.000 & 4.163 & 1.980 \\
& P4 & 36.392 & 25.000 & 9.729 & 1.778 \\
2 & P5 & 28.664 & 22.000 & 3.758 & 1.895 \\
& P6 & 23.056 & 15.000 & 4.692 & 2.626 \\
\hline
\end{tabular}

OTU: unidad taxonómica operativa. 
La comunidad 2 presentó la mayor cantidad de secuencias, tanto iniciales como finales, seguida de la comunidad 1. Cabe destacar que el la comunidad 1 mostró la mayor cantidad de secuencias de microorganismos no catalogados científicamente (no clasificados), a pesar de presentar un menor número de secuencias, tanto iniciales como finales. De la misma forma, el número de agrupamiento de géneros (OTU) fue mayor para la comunidad 1, a pesar de presentar un menor número de secuencias, seguido de la comunidad 2.

\section{Curva de rarefacción}

Una curva de rarefacción consiste en calcular el número esperado de especies (diversidad) en cada muestra, para un tamaño de muestra patrón. Estas curvas permiten extrapolar la relación de OTU en función del número de secuencias e indican el efecto del esfuerzo realizado en la secuenciación, para estimar el valor máximo de OTU en un nivel filogenético. La obtención de una curva de este tipo permite la comparación de muestras, aun con intensidades de muestras diferentes (Ferreira, 2011).

Los datos para la construcción de las curvas de rarefacción de las comunidades de estudio en esta investigación, fueron los datos generados a partir del programa Mothur con $97 \%$ de similaridad, considerando que dos secuencias pertenecen a la misma OTU si presentan una distancia p menor al $3 \%$. En la Figura 5 puede visualizarse la curva de rarefacción para las dos comunidades analizadas. Considerando el número de secuencias para las dos comunidades, puede observarse que aunque se aumente el número de estas, la tendencia de las curvas es hacia la estabilidad.

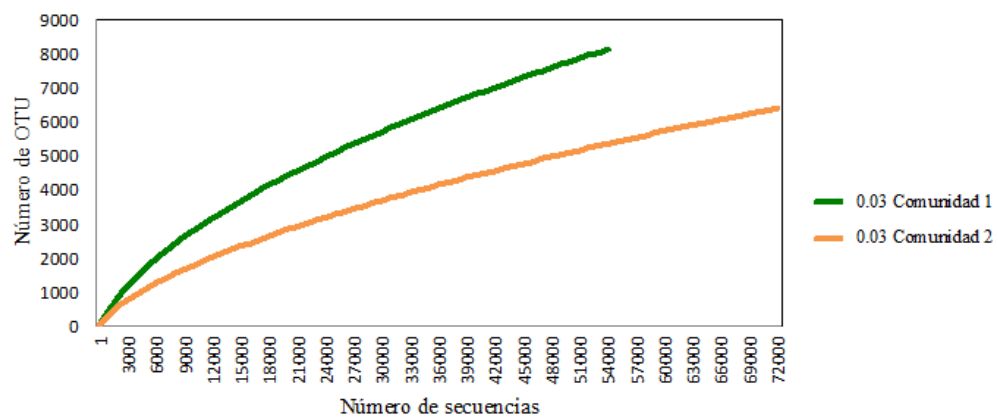

Figura 5. Curvas de rarefacción para las comunidades del riachuelo con vertido continuo de efluentes de hidrocarburos. OTU: unidad taxonómica operativa. Comunidad 1: antes del vertido, comunidad 2: después del vertido.

Este resultado indica que el número de secuencias realizadas en cada biblioteca, fue relativamente suficiente para describir la diversidad presente en cada comunidad analizada. Asimismo, puede visualizarse que la diversidad fue mayor para comunidad 1, o sea la cantidad de OTU fue mayor con relación a la comunidad 2.

\section{Índices de riqueza y diversidad}

La estimación de los diferentes índices de riqueza y diversidad bacteriana fue realizada mediante aplicación del programa Mothur (Schloss, 2009), el cual considera los índices específicos de Chao1 y Ace para estimar la riqueza y los índices de Shannon y Simpson para la diversidad (97 \% similitud). Los datos de los índices de riqueza y diversidad, basados en las OTU, se encuentran en la Tabla 4.

Tabla 4. Índices de riqueza y diversidad (97\% similitud) para las comunidades del riachuelo con vertido continuo de efluentes de hidrocarburos. Comunidad 1: antes del vertido, comunidad 2: después del vertido.

\begin{tabular}{|c|c|c|c|c|c|c|c|}
\hline \multirow{2}{*}{$\begin{array}{l}\text { Número de } \\
\text { comunidad }\end{array}$} & \multirow{2}{*}{$\begin{array}{c}\text { Código } \\
\text { muestra }\end{array}$} & \multirow{2}{*}{$\begin{array}{c}\mathrm{N}^{\circ} \text { secuencias } \\
\text { finales }\end{array}$} & \multirow{2}{*}{$\begin{array}{l}\text { No. } \\
\text { UTO }\end{array}$} & \multicolumn{2}{|c|}{ Índices de riqueza } & \multicolumn{2}{|c|}{ Índices de diversidad } \\
\hline & & & & Chao1 & Ace & Shannon & Simpson \\
\hline & P1 & 10.000 & 2.578 & 6.169 & 9.829 & 6,62 & 0,005 \\
\hline 1 & $\begin{array}{l}\text { P2 } \\
\text { P3 } \\
\text { P4 }\end{array}$ & $\begin{array}{c}4.000 \\
13.000 \\
25.000\end{array}$ & $\begin{array}{l}1.109 \\
1.980 \\
3.709\end{array}$ & $\begin{array}{l}3.051 \\
4.353 \\
7.996\end{array}$ & $\begin{array}{c}4.823 \\
6.636 \\
12.083\end{array}$ & $\begin{array}{l}5,52 \\
5,67 \\
6,01\end{array}$ & $\begin{array}{l}0,025 \\
0,016 \\
0,012\end{array}$ \\
\hline & P5 & 22.000 & 1.778 & 4.390 & 7.991 & 3,02 & 0,363 \\
\hline 2 & $\begin{array}{l}\text { P6 } \\
\text { P7 } \\
\text { P8 }\end{array}$ & $\begin{array}{l}15.000 \\
21.000 \\
12.000\end{array}$ & $\begin{array}{l}1.895 \\
2.626 \\
1.634\end{array}$ & $\begin{array}{l}4.760 \\
5.975 \\
3.790\end{array}$ & $\begin{array}{l}7.654 \\
9.407 \\
6.293 \\
\end{array}$ & $\begin{array}{l}5,44 \\
5,20 \\
4,83\end{array}$ & $\begin{array}{l}0,020 \\
0,057 \\
0,083\end{array}$ \\
\hline
\end{tabular}

OTU: unidad taxonómica operativa. 
El índice de estimación de riqueza Chao1 es basado en la abundancia de OTU (únicas y raras), para estimar la riqueza de una populación de tamaño desconocido (Schloss, 2009). Por otra parte, el índice de Ace, es un método no paramétrico para estimar el número de especies existentes en una comunidad microbiana, y se define como la suma de las probabilidades de las especies observadas. El método Ace divide las frecuencias observadas en abundantes y grupos raros (Kim et al., 2017).

En los resultados observados en la Tabla 4, específicamente con relación a los índices de riqueza, Chao1 y Ace, algunos valores presentan disminución y otros presentan incremento entre las muestras de la misma comunidad, así como entre muestras de las dos comunidades analizadas. Para realizar un análisis de variación porcentual de estos índices, se realizó la comparación de los resultados en los diferentes puntos de colecta de las muestras (a 50 y 80 m aguas arriba y aguas abajo después del vertido de efluentes, tanto en el centro y en la orilla del riachuelo, respectivamente). En la Tabla 5 puede visualizarse el análisis comparativo de la variación porcentual en los índices de riqueza.

Tabla 5. Análisis de variación porcentual de los índices de riqueza para las comunidades del riachuelo con vertido continuo de efluentes de hidrocarburos. Comunidad 1: antes del vertido, comunidad 2: después del vertido.

\begin{tabular}{|c|c|c|c|c|c|c|c|}
\hline \multirow[t]{2}{*}{ Localidad } & \multicolumn{3}{|c|}{ Puntos de muestreo en el riachuelo } & \multicolumn{4}{|c|}{$\begin{array}{c}\text { Índice de riqueza } \\
\text { Variación porcentual (\%) }\end{array}$} \\
\hline & Comunidad 1 & Vs. & Comunidad 2 & \multicolumn{2}{|c|}{ Chao1 } & \multicolumn{2}{|c|}{ Ace } \\
\hline Centro de riachuelo & $\mathrm{P} 1$ & $\cdots$ & P5 & 28,8 & $\hat{\mathrm{e}}$ & 18,6 & $\hat{\mathrm{e}}$ \\
\hline $\begin{array}{l}\text { Orilla de riachuelo a } \\
50 \mathrm{~m}\end{array}$ & P2 & $\cdots$ & P6 & 56,0 & é & 58,7 & é \\
\hline Centro de riachuelo & P3 & $\cdots$ & P7 & 37,2 & é & 41,7 & é \\
\hline $\begin{array}{l}\text { Orilla de riachuelo a } \\
80 \mathrm{~m}\end{array}$ & P4 & $\ldots$ & P8 & 52,6 & $\hat{\mathrm{e}}$ & 47,9 & $\hat{\mathrm{e}}$ \\
\hline
\end{tabular}

ê: disminución, é: incremento.

Al comparar los resultados de la muestra P1 con la muestra P5 (Tabla 5), las cuales están equidistantes a $50 \mathrm{~m}$ del punto de vertido de efluentes, en el centro del riachuelo, pudo observarse la disminución del 28,8 \% en el índice Chao1 y una disminución del 18,6 \% en el índice Ace. Un comportamiento similar se obtiene al comparar los puntos de muestreo P4 y P8. Sin embargo, entre los puntos P2 y P6, y entre P3 y P7, se observó un incremento de 56,0 y 37,2 \% en el índice Chao1 y de 58,7 y 41,7 \% en el índice Ace, respectivamente. Por lo tanto, en función de esta variación porcentual (disminución e incremento) de losíndices de Chao1 y Ace, no se puede afirmar que la riqueza bacteriana sea mayor o menor en la comunidad 1 con relación a la comunidad 2.

Por otra parte, el índice de Shannon mide la diversidad y es utilizado en situaciones en las que una comunidad entera no puede ser inventariada. Su cálculo lleva en consideración que las especies presentan abundancias diferentes (Rodrigues, 2011). En el resultado de esta investigación (Tabla 4), este índice presentó una disminución en todos los valores de la comunidad 2, con relación a la comunidad 1, indicando que la comunidad 1 posee una mayor diversidad. Adicionalmente, el índice de Simpson mide la dominancia dentro de una comunidad y la sensibilidad a los cambios en las especies abundantes. Es útil para el monitoreo ambiental, el cual mide la variación de las especies más abundantes por alguna perturbación. Los valores de la diversidad, según índice Simpson, se encuentran dentro de una escala de 0 a 1 ; siendo mayor cuando se aproxima a uno, lo que indicará mayor dominancia y, por consecuencia, menor diversidad (Ñique, 2010). Los resultados de la diversidad, según el índice de Simpson (Tabla 4), fueron mayores (más próximos a 1) en la comunidad 2 , lo que indica la existencia de una mayor dominancia de especies en la comunidad 2, pero con disminución en la diversidad. Por lo tanto, la comunidad 1, presentó una diversidad mayor con relación a la comunidad 2.

\section{Análisis estadístico}

El análisis AMOVA (generado por el programa Mothur) es una prueba estadística análogo al ANOVA (análisis de varianza), y consiste en realizar el análisis molecular de la varianza. Este método se usa ampliamente en genética de poblaciones para probar la hipótesis de que la diversidad genética dentro de dos poblaciones, no es significativamente diferente de la que resultaría de la mezcla de ambas poblaciones (Neves, 2013). Los resultados del AMOVA generados por el programa Mothur, se encuentran en la Tabla 6. 
Tabla 6. Resultados del AMOVA (programa Mothur) para las comunidades del riachuelo con vertido continuo de efluentes de hidrocarburos. Comunidad 1: antes del vertido, comunidad 2: después del vertido.

\begin{tabular}{cccc}
\hline Variación & & AMOVA & Total \\
\hline Comunidad 1 vs. Comunidad 2 & Entre los grupos & Dentro de los grupos & 138,502 \\
SS & 127,569 & 6 & 7 \\
df & 1 & 0,230836 & \\
MS & 127,569 & & \\
Fs: & 552,637 & & \\
$p_{\text {value }}: 0,023$ & & & \\
\hline
\end{tabular}

SS: suma de los cuadrados entre las dos comunidades; df: grados de libertad; MS: cuadrado medio entre las dos comunidades; Fs: valor de F para $\alpha=0,01 ; p_{\text {value }}$ : probabilidad.

Como puede observarse en la comparación global entre las dos comunidades de estudio (Tabla 6), el resultado refleja que no existe diferencia significativa entre ambas comunidades ( $\left.\mathrm{p}_{\text {value }}:>0,001\right)$. Adicionalmente, el análisis de LIBSHUFF (Mothur) procura evaluar si las muestras (bibliotecas) de cada una de las comunidades analizadas, poseen la misma estructura genética (Schloss, 2009). Los resultados generados del análisis LIBSHUFF, se presentan en la Tabla 7.

Los resultados muestran que no existe diferencia significativa en la estructura genética entre las muestras de cada una de las dos comunidades analizadas $(p>0,008$; valor de $p$ calculado después de la corrección de Bonferroni, $p=$ significancia/número de comparaciones, $p=0,1 / 12$ ).

Tabla 7. Resultados del análisis LIBSHUFF (programa Mothur) para las comunidades del riachuelo con vertido continuo de efluentes de hidrocarburos. Comunidad 1: antes del vertido, comunidad 2: después del vertido.

\begin{tabular}{cccc}
\hline & Comunidad 1 & \multicolumn{2}{c}{ Comunidad 2 } \\
\hline $\begin{array}{c}\text { Comparación } \\
\text { entre muestras } \\
\text { (dCXYScore) }\end{array}$ & $\begin{array}{c}\text { Probabilidad } \\
\text { P } \\
\text { (significancia 10 \%) }\end{array}$ & $\begin{array}{c}\text { Comparación } \\
\text { entre muestras } \\
\text { (dCXYScore) }\end{array}$ & $\begin{array}{c}\text { Probabilidad } \\
\text { P }\end{array}$ \\
\hline P1-P2 & 0,712 & P5-P6 & 0,798 \\
P2-P1 & 0,712 & P6-P5ificancia 10 \%) \\
P1-P3 & 0,849 & P5-P7 & 0,798 \\
P3-P1 & 0,849 & P7-P5 & 0,520 \\
P1-P4 & 0,790 & P5-P8 & 0,520 \\
P4-P1 & 0,790 & P8-P5 & 0,390 \\
P2-P3 & 0,888 & P6-P7 & 0,390 \\
P3-P2 & 0,888 & P7-P6 & 0,437 \\
P2-P4 & 0,768 & P6-P8 & 0,437 \\
P4-P2 & 0,768 & P8-P6 & 0,551 \\
P3-P4 & 0,854 & P7-P8 & 0,551 \\
P4-P3 & 0,854 & P8-P7 & 0,130 \\
\hline
\end{tabular}

\section{Conclusiones}

En la comunidad 1, el filo dominante fue Acidobacteria con la clase Deltaproteobacteria, siendo los géneros Gp3 y Geobacter los preponderantes en esta comunidad de estudio. Con relación a la comunidad 2, el filo Proteobacteria, la clase Betaproteobacteria y los géneros Geobacter y $G p 3$, fueron los predominantes. 
Una parte de los microorganismos resultaron "no clasificados" (no catalogados científicamente), aproximadamente $31 \%$ en comunidad 1 y $25 \%$ en comunidad 2 . Aproximadamente $15 \%$ de todos los géneros bacterianos identificados fueron catalogados como biorremediadores de ambientes contaminados.

Los índices de riqueza presentaron variación porcentual (disminución e incremento) en ambas comunidades, lo cual no permite definir en cuál de las dos fue mayor la riqueza. Con respecto al índice de diversidad, los resultados indican que la diversidad bacteriana en el comunidad 1, fue más abundante con relación a la comunidad 2 . Sin embargo, estadísticamente se comprobó que no existe diferencia significativa en la comparación global entre ambas comunidades $(p>0,001)$. Asimismo, los resultados mostraron la misma estructura genética entre las muestras de cada comunidad analizada separadamente $(p>0,008)$.

Los resultados obtenidos representan un gran desafío para la exploración biotecnológica y comprensión de la composición, riqueza y diversidad de la comunidad bacteriana existente en este ecosistema, así como un gran potencial para identificar y clasificar nuevos grupos taxonómicos.

\section{Agradecimientos}

Este trabajo fue posible gracias al apoyo del programa multi-institucional de post graduación en biotecnología (PPGBIOTEC) de la Universidad Federal del Amazonas (UFAM), por el aporte de recursos humanos y económicos para realizar todos los análisis respectivos. Igualmente se agradece a la Gerencia de Seguridad, Medio Ambiente y Salud (SMS) de la empresa Petrobras-AM, por la logística y apoyo para realizar las diversas visitas de campo para recolectar muestras de estudio. Así mismo, se agradece a la Facultad de Tecnología, Departamento de Ingeniería de Producción de la Universidad Federal del Amazonas UFAM, por promover la publicación de este artículo.

\section{Referencias bibliográficas}

Alvaredo, D. A. P. (2009). Prospecção gênica e diversidade bacteriana de um consórcio degradador de óleo diesel. Dissertação Faculda de de Ciências Agrárias e Veterinárias. Jaboticabal, SP. Brasil.

Amann, R. I., Ludwig, W., Schleifer, K. H. (1995). Phylogenetic identification and in situ detection of individual microbial cells without cultivation. Microbiology Review, 59, 143-169.

Atlas, R. M., Cerniglia, C. E. (1995). Bioremediation of petroleum pollutants. Bioscience, 45, 332-338.

Bicudo, C. E. de M. (2004). Taxonomia. Biota Neotropica, 4(1), I-IIp.

Cannavan, F. S. (2007). Diversidade das comunidades bacterianas em solos de Terra Preta Antropogênica da Amazônia Central e Oriental Piracicaba. Dissertação. Escola Superior de Agricultura "Luiz de Queiroz". Universidade de São Paulo, Piracicaba, SP. Brasil.

CONAMA. (2005). Capítulo III. Das condições e padrões de qualidade das águas. Conselho Nacional do Meio Ambiente. Resolução No. 357, de 17 de março de 2005. Publicada no DOU No 053, de 18/03/2005, Brasilia.

Etto, R. M. (2011). Comunidades procarióticas das turfeiras dos campos de altitude paranaenses. Tese. Universidade Federal do Paraná, Curitiba. Brasil.

Ferreira, A. J. (2011). Diversidade e estrutura da comunidade bacteriana associada às armadilhas da planta carnívora Utricularia gibba (Lentibulariaceae) e o ambiente aquático. Dissertação. Instituto de Ciências Biomédicas, Universidade de São Paulo. SP. Brasil.

Hatamono, M., Imachi H., Yashiro Y., Ohashi A., Harada H. (2007). Diversity of anaerobic microorganisms involved in long-chain fatty acid degradation in methanogenic sludges as revealed by RNA-based stable isotope probing. Applied and Environmental Microbiology, 73(13), 4119-4127.

Hwang, C., Copeland, A., Lucas, S., Lapidus, A., Barry, K., Glavina Del Rio T., Dalin, E., Tice, H., Pitluck, S., Sims, D., Brettin, T., 
Bruce, D. C., Detter, J. C., Han, C. S., Schmutz, J., Larimer, F. W., Land, M. L., Hauser, L. J., Kyrpides, N., Lykidis, A., Richardson, P., Belieav, A., Sanford, R. A., Löeffler, F. E., Fields, M. W. (2015). Complete genome sequence of Anaeromyxobacter sp. FW109-5, an anaerobic, metal-reducing bacterium isolated from a contaminated subsurface. Environment. Genome Announcements, 3(1), e01449-14.

Jacques, R; Bento, F. M; Antoniolli, Z. I., Camargo, F. A. (2007). Biorremediação de solos contaminados com hidrocarbonetos aromáticos policíclicos. Ciência rural, 37(4), 1192-1201.

Jin J., Wu G., Guan Y. (2015). Effect of bacterial communities on the formation of cast iron corrosion tubercles in reclaimed water. Water Res., 71, 207-218.

Kielak, A., Pijl, A., Van-Veen, J., Kowalchuk, G. (2008). Differences in vegetation composition and plant species identity lead to only minor changes in soil-borne microbial communities in a former arable field. FEMS. Microbiology Ecology, 63(3), 372382 .

Kim, B. R., Jiwon, S., Guevarra, R, Lee, J., Kim, D., Seol, K-H., Lee, J. H, Kim, H. B. Isaacson, R. (2017). Deciphering diversity indices for a better understanding of microbial communities. Microbiol. Biotechnol., 27(12), 2089-2093.

Kleinsteuber, S., Schleinitz K. M., Vogt C. (2012). Key players and team play: anaerobic microbial communities in hydrocarboncontaminated aquifers. Applied Microbiology and Biotechnology, 94(4), 851-873.

Mandri, T., Lin, J. (2007). Isolation and characterization of en Kwazulu-Natal, South Africa. African Journal of Biotechnology, 6(1), 023-027.

Neves, R. O. (2013). Caracterização da microbiota bacteriana da água do Rio Negro em diferentes períodos sazonais. Dissertação. Universidade Federal do Amazonas UFAM, AM, Brasil.

Nevin, K., Lovley, D. R. (2002). Mechanisms for accessing insoluble Fe(III) oxide during dissimilatory Fe(III) reduction by Geothrix fermentans. Applied and Environmental Microbiology, 68(5), 2294-2299.

Ñique, M. (2010). Biodiversidad: Clasificación y cuantificación. Universidad Nacional Agrária de la Selva. Tingo María, Perú.

Nishimura, Y., Nagata, T. (2007). Alphaproteobacterial dominance in a large mesotrophic lake (Lake Biwa, Japan). Aquatic Microbial Ecology, 48, 231-240.

Nústez C., Paredes D., Cubillos. J. (2014). Biorremediación para la degradación de hidrocarburos totales presentes en los sedimentos de una estación de servicio de combustible. Rev. Téc. Ing. Univ. Zulia, 37(1), 20-28.

Parmar S., Sharma, V. K., Kumar, J. (2019). Aplication of molecular and sequencing techniques in analysis of microbial diversity in agroecosystem. In: Microbial Genomics in Sustainable Agroecosystems, Ed. Tripathi, V., Kumar, P., Tripathi, P., Kishore, A., Kamle. M. Singapore: Springer.

Peixoto, J. C., Leomil L., Souza J. V., Peixoto F.B. S., Astolfi-Filho S. (2011). Comparison of bacterial communities in the Solimões and Negro River tributaries of the Amazon River based on small subunit rRNA gene sequences. Genetics and Molecular Research, 10(4), 3783-3793.

Pereyra, L. P., Hiibel, S. R., Prieto M. V., Reardon, K. F., Pruden, A. (2010). Detection and quantification of functional genes of cellulose degrading, fermentative, and sulfate-reducing Bacteria and methanogenic Archaea. Applied and Environmental Microbiology, 76(7), 2192-2202.

Ramos, D. T. (2013). Bioestimulação de processos metanogênicos com acetato de amônio para degradação acelerada de hidrocarbonetos de petróleo em águas subterrâneas contaminadas com diesel B20. Tese. Universidade Federal de Santa Catarina. Florianópolis, SC. Brasil.

Rodrigues, T. B. (2011). Diversidade metagênomica microbiana de biomas terrestres e marinhos. Tese. Universidade Federal 
de Rio de Janeiro, RJ. Brasil.

Schloss, P. D. (2009). A high-throughput DNA sequence aligner for microbial ecology studies. PLoS ONE, 4, 8230.

Seo, J. S., Keum Y. S., Li, Q. X. (2009). Bacterial degradation of aromatic compounds. International Journal of Environmental Research, and Public Health, 6, 278-309.

Silva, C. L. V., Silva A. L. S, De Medeiros, S. R. B., Lima L. F., Blaha C. A. G. (2007). Detecção de bacterias redutoras de Fe (III), em mangue da bacia petrolífera Portiguar com potencial biodegradador de petróleo. $4^{\circ}$ PDPETRO. Campinas, SP. Brasil.

Sun, W., Sun X., Cuples A. M. (2012). Anaerobic methyl tert-butyl ether-degrading microorganisms identified in wastewater treatment plant samples by stable isotope probing. Applied and Environmental Microbiology, 78(8), 2973-2980.

Torsvik, V., Salte K., Sorheim, R., Goksoyr, J. (1990). Comparison of phenotypic diversity and DNA heterogeneity in a population of soil bacteria. Appl. Environ. Microbiol., 56(3), 776-781.

Toyama, D. (2012). Análise da diversidade microbiana aquática em rios e lagos da região amazônica. Dissertação. Universidade de São Paulo, SP. Brasil.

Xavier, J. C., Costa, P. E. S., Hissa, D. C, Melo, V. M. M, Lima, M. G. S., Coutinho, H., Verde, L. C. L (2019). Evaluation of the microbial diversity and heavy metal resistance genes of a microbial community on contaminated environment. Applied Geochemistry, 105, 1-6.

Zwart, G., Zwart, G., Crump, B. C., Agterveld, M. P. K. V., Hagen, F., Han, S. K. (2002). Typical freshwater bacteria: an analysis of available 16S rRNA gene sequences from plankton of lakes and rivers. Aquatic Microbiol. Ecol., 28, 141-155. 


\section{REVISTA TECNICA}

DE LA FACULTAD DE INGENIERIA

UNIVERSIDAD DEL ZULIA

Vol. 44. №2, Mayo - Agosto, 2021

Esta revista fue editada en formato digital y publicada en Abril de 2021, por el Fondo Editorial Serbiluz, Universidad del Zulia. Maracaibo-Venezuela

www.luz.edu.ve

www.serbi.luz.edu.ve

www.produccioncientificaluz.org 